\title{
Global Electroweak Fits in the SM and MSSM
}

\author{
Wim de Boer* and Christian Sander \\ IEKP, Univ. of Karlsruhe, Postfach 6980, 76128 Karlsruhe, Germany \\ E-mail: 'wim.de.boer@cern.ch'
}

ABstract: A global statistical $\chi^{2}$ analysis of all electroweak data including new data on the anomalous magnetic moment of the muon and the $b \rightarrow X_{s} \gamma$ decay rate in both the SM and the MSSM has been performed. The total $\chi^{2}$ of the MSSM is better than in the SM, mainly because of the W-mass, $a_{\mu}$ and $b \rightarrow X_{s} \gamma$, although the total probability is similar in both models due to the larger number of parameters in the MSSM.

\section{Introduction}

A few year ago a complete electroweak fit program including all possible supersymmetric corrections in the Minimal Supersymmetric Model (MSSM) was developed, mainly to investigate the so-called $R_{b}$ deviation of the Standard Model (SM) [i, $]_{1}^{1}$. At present $R_{b}$ shows no significant deviation from the SM, but the present total $\chi^{2}$ of all electroweak data is not excellent[2i]. In addition, if the new measurements of the anomalous magnetic moment [3i] and $b \rightarrow X_{s} \gamma[\bar{i}]$ are included, the SM fit becomes worse. It is the purpose of the present paper to include these new measurements in the SM fits and compare the SM fit with the MSSM fit.

\section{Experimental Data}

A summary of the most recent electroweak data from colliders can be found in the report from the Electroweak Working Group (EWWG) [i 2$]$. As mentioned above, we included in addition the anomalous magnetic moment of the muon $a_{\mu}$, which was recently determined by the E821 collaboration from a measurement of g-2 using the polarization in the decays of muons in a muon storage ring. They found $a_{\mu}$ to be slightly above the SM prediction, the difference being $\Delta a_{\mu}=a_{\mu}^{\text {exp }}-a_{\mu}^{t h}=(43 \pm 16) \cdot 10^{-10}\left[\begin{array}{l}3 \\ {[3}\end{array}\right]$. However, the largest SM contribution to $a_{\mu}$ comes from the radiative loop corrections to the electromagnetic coupling constant, which consist of an hadronic and leptonic part. The hadronic part cannot be calculated at low $Q^{2}$, since it is dominated by non-perturbative contributions of resonances to the photon self-energy. This part is determined from the low energy hadronic

\footnotetext{
${ }^{*}$ Speaker.
} 

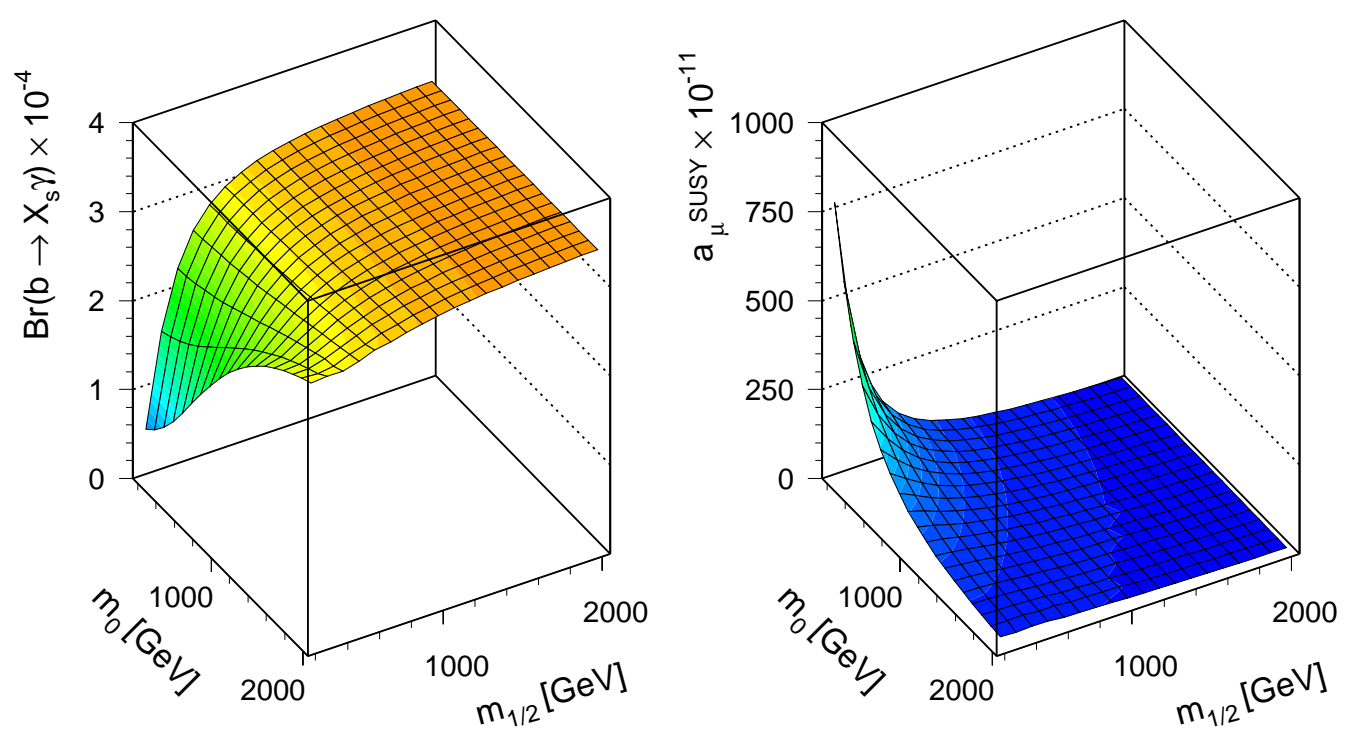

Figure 1: The values of $b \rightarrow X_{s} \gamma$ and $a_{\mu}^{S U S Y}$ in the $m_{0}, m_{1 / 2}$ plane for positive $\mu$ and $\tan \beta=35$ to be compared with experimental data $b \rightarrow X_{s} \gamma=(3.23 \pm 0.41) \cdot 10^{-4}$ and $a_{\mu}^{S U S Y}=(333 \pm 173)$. $10^{-11}$. One can see that both $b \rightarrow X_{s} \gamma$ and $a_{\mu}^{S U S Y}$ prefer relatively light sparticles.

cross section in $e^{+} e^{-}$annihilation. In Ref. [3ian $]$the newest data on $e^{+} e^{-}$annihilation from the Beijing accelerator were not yet considered. A recent evaluation including the newest $e^{+} e^{-}$annihilation data and assuming CVC reduces $\Delta a_{\mu}$ to $(33 \pm 17) \cdot 10^{-10}\left[\begin{array}{l}4 \\ -1\end{array}\right]$. We will conservatively use this reduced value in the fits below.

The most popular explanation for contributions outside the SM is given in the frame-

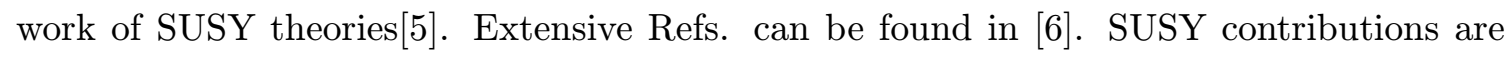
also expected to affect the $b \rightarrow X_{s} \gamma$ rate, for which the most recent world average is: $b \rightarrow X_{s} \gamma=(3.23 \pm 0.41) \times 10^{-4}$. This value is dominated by the recently published results from CLEO $\left(\left(3.21 \pm 0.43_{\text {stat }} \pm 0.27_{\text {sys }} \pm 0.14_{\text {mod }}\right) \times 10^{-4}\right)\left[\overline{z_{1}}\right]$, which is consistent

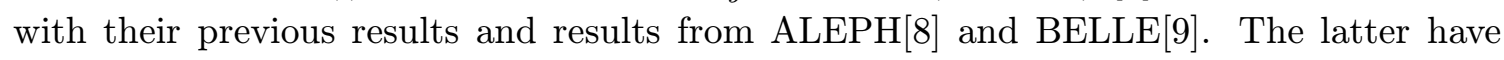
considerably larger errors. Also the published CLEO result has larger errors than the

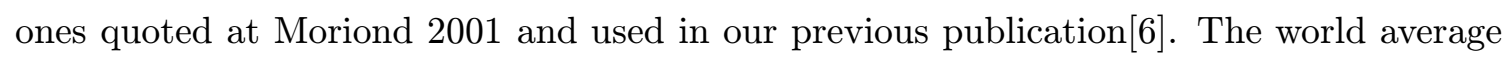
is slightly below, but consistent with a recent SM prediction by Gambino and Misiak of $(3.73 \pm 0.30) \times 10^{-4}\left[{ }_{1}^{[} \bar{O}_{1}\right]$. This value is somewhat higher than previous values, since it uses the running mass for the charm quark in the loops, while keeping the pole mass for the b-quark in the external lines. This gives an additional uncertainty, but the authors found a reduced scale dependence. In our present analysis we conservatively keep a theoretical error of $\pm 0.40 \times 10^{-4}$, but use $m_{c}(\mu) / m_{b}=0.22$. This is not critical, since with the present large experimental error $b \rightarrow X_{s} \gamma$ hardly constrains the present fit. It is interesting to note, that the positive sign of $\mu$ and the relatively light sparticle spectrum, as required by $a_{\mu}$, yield indeed a value of $b \rightarrow X_{s} \gamma$ slightly below the SM value, as shown in Fig. 


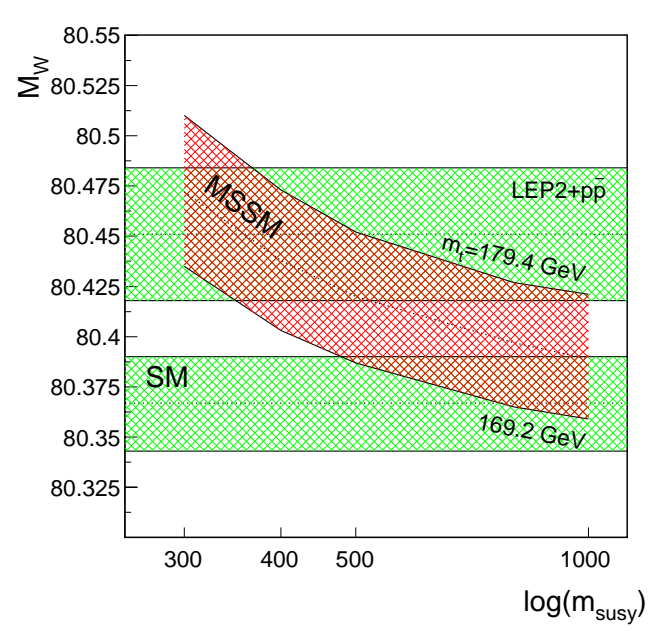

Figure 2: $W$ mass versus sparticle masses, assuming all sparticles have the same mass. The horizontal bands represent the SM prediction from LEP I data and the direct measurements from the LEP II and $p \bar{p}$. The curved band is the MSSM prediction for the case that all sparticles have a given mass $m_{\text {susy }}$. Its width is determined by the uncertainty from the top mass.

The value of $M_{W}$ becomes higher in the MSSM than in the SM, as shown in Fig. 12, in agreement with the direct measurements of $M_{W}$ at LEP II and $p \bar{p}$.

None of these measurements, $a_{\mu}, b \rightarrow$ $X_{s} \gamma$ and $M_{W}$, show by itself a significant deviation from the SM, but since they all point to supersymmetric contributions, it is interesting to compare a global fit of all data in the SM and MSSM.

\section{Fit Results}

All electroweak variables were calculated in the SM using ZFITTER6.11[1] MSSM using MSSMFITTER[י][1]. The deviation between data and theoretical prediction in the SM and MSSM are shown in Fig. 3. Clearly, the largest deviations occur in the forward-backward asymmetry $A_{F B}^{b}$ for bquarks and the left-right asymmetry, as measured with the polarized electron beam at SLAC. Both can be translated into a measurement of the electroweak mixing angle, which than turns out to be $3 \sigma$ apart [2i]. In the MSSM the situation does not improve. Since there is no preference for any of the data, we followed the procedure from the Particle Data Group to rescale the errors of $A_{F B}^{b}$ and $A_{L R}$ in such a way that their $\chi^{2}$ contributions are about one. This hardly influences any of the other variables, as shown on the right hand side of Fig. 13 , but increases the probability from below $5 \%$ to $25 \%(36 \%)$ in the SM (MSSM). Also the fitted parameters for the SM and MSSM, shown in Tables 'ili-1, and a critical issue. The fact that the SM fit prefers a Higgs mass slightly below the experimental limit is not an issue either, since requiring the Higgs mass to be above the limit from the direct searches $\left(m_{h}>114 \mathrm{GeV}\right.$, [1] ${ }_{1}^{1}$ in $)$ only causes a minor shift in the top mass in the fit (see Table $\left.\underline{1}_{1}^{\overline{1}}\right)$. The $\chi^{2} /$ d.o.f. in the MSSM is better than in the SM (14.2/13 for MSSM versus $20.5 / 17$ for SM), mainly because of $a_{\mu}, b \rightarrow X_{s} \gamma$ and $M_{W}$ (see Fig. 商), but the probabilities are comparable due to the larger number of parameters in the MSSM as shown in the tables. The MSSM fits are not very sensitive to $\tan \beta$, provided it is large.

\section{Conclusion}

It has been shown that a SM electroweak fit including the anomalous magnetic moment and $b \rightarrow X_{s} \gamma$ yields a probability below $5 \%$, even with conservative error estimates. This probability is improved in the MSSM, mainly because of $a_{\mu}, b \rightarrow X_{s} \gamma$ adn $M_{W}$. However, in both cases the $3 \sigma$ discrepancy in $\sin ^{2} \theta_{W}$ from the $A_{F B}^{b}$ and $A_{L R}$ is the main source for 

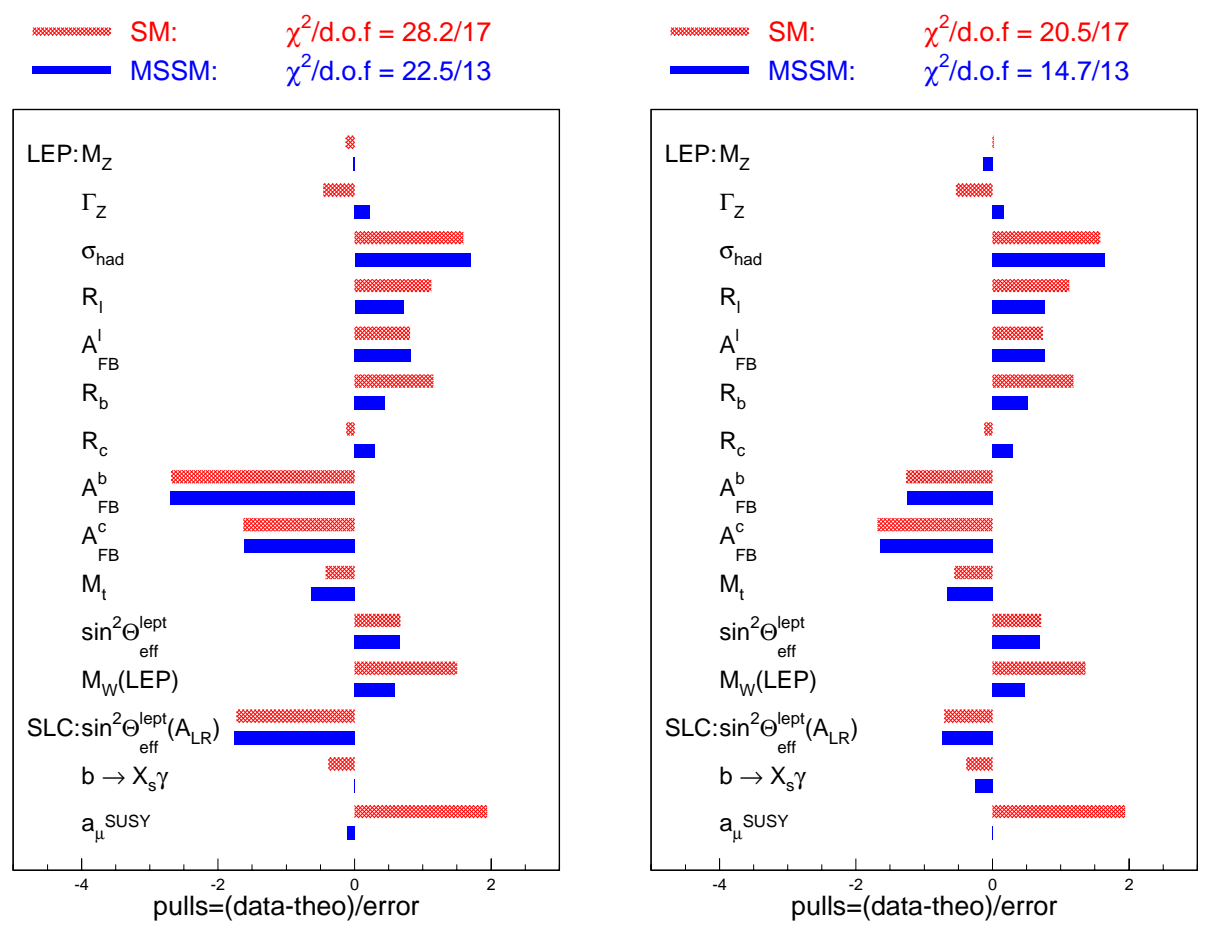

Figure 3: The pulls of the electroweak data for the SM and MSSM

\begin{tabular}{|c||c|c|c|}
\hline Parameter & SM & SM + higgs & SM + higgs + rescaled \\
\hline \hline$M_{Z}[\mathrm{GeV}]$ & $91.1877(21)$ & $91.1877(21)$ & $91.1875(21)$ \\
$m_{t}[\mathrm{GeV}]$ & $175.4(3.8)$ & $176.5(3.1)$ & $177.2(3.2)$ \\
$m_{h}[\mathrm{GeV}]$ & $99_{-43}^{+53}$ & 114.1 & 114.1 \\
$\alpha_{s}\left(M_{Z}\right)$ & $0.1186(27)$ & $0.1185(26)$ & $0.1184(25)$ \\
$\Delta \alpha_{\text {had }}^{(5)}\left(M_{Z}\right)$ & $0.02764(33)$ & $0.02763(31)$ & $0.02757(33)$ \\
\hline \hline $\sin ^{2} \theta(\overline{\mathrm{MS}})$ & $0.23140(15)$ & $0.23145(10)$ & $0.23141(12)$ \\
$\sin ^{2} \theta_{\text {eff }}^{\text {lept }}$ & $0.23140(13)$ & $0.23143(11)$ & $0.23139(13)$ \\
$M_{W}$ & $80.393(18)$ & $80.392(18)$ & $80.398(19)$ \\
\hline \hline$\chi^{2} /$ d.o.f. & $28.1 / 17$ & $28.2 / 17$ & $20.5 / 17$ \\
Probability & $4.4 \%$ & $4.3 \%$ & $24.9 \%$ \\
\hline
\end{tabular}

Table 1: SM fit parameters. The first column uses the input data discussed in the text, which yields as most probable Higgs mass $m_{h}=99 \mathrm{GeV}$; the second column requires the Higgs mass to be above the experimental limit of $114 \mathrm{GeV}$ at $95 \%$ C.L. is below $5 \%$, which is mainly caused by the $3 \sigma$ discrepancy between the $\sin ^{2} \theta_{W}$ values from $A_{F B}^{b}$ and $A_{L R}$. In the last column these errors have been rescaled (see text). The electroweak mixing angles and $M_{W}$ are derived quantities, not fitted parameters.

the low probability. Since at present no arguments to doubt any of the measurements can be found, we tested the Particle Data Group's procedure to rescale the errors of these two measurements by the corresponding pull. This yields considerably improved fits, both in the SM and MSSM, without significant changes in the fitted parameters. 


\begin{tabular}{|c|c|c|c|c|c|c|}
\hline \multicolumn{6}{|c|}{ SUSY Parameters } \\
\hline & \multicolumn{7}{|c|}{ rescaled } \\
\hline Symbol & $\tan \beta=35$ & $\tan \beta=20$ & $\tan \beta=50$ & $\tan \beta=35$ & $\tan \beta=20$ & $\tan \beta=50$ \\
\hline$m_{t}[\mathrm{GeV}]$ & 177.5 & 176.0 & 176.8 & 177.7 & 177.0 & 176.8 \\
$\alpha_{s}$ & 0.1178 & 0.1179 & 0.1178 & 0.1177 & 0.1181 & 0.1184 \\
$m_{\tilde{t}_{1}}[\mathrm{GeV}]$ & 1099 & 956 & 968 & 799 & 825 & 690 \\
$m_{\tilde{t}_{2}}[\mathrm{GeV}]$ & 213 & 255 & 251 & 268 & 279 & 297 \\
$m_{\tilde{b}_{1}}[\mathrm{GeV}]$ & 1087 & 945 & 954 & 783 & 810 & 672 \\
$m_{\tilde{b}_{2}}[\mathrm{GeV}]$ & 1451 & 921 & 1213 & 1140 & 1170 & 1867 \\
$m_{\tilde{q}}[\mathrm{GeV}]$ & 1087 & 945 & 954 & 783 & 810 & 672 \\
$m_{\tilde{l}}[\mathrm{GeV}]$ & 575 & 385 & 894 & 598 & 393 & 716 \\
$m_{\tilde{\chi}_{1}^{ \pm}}[\mathrm{GeV}]$ & 221 & 220 & 221 & 221 & 220 & 222 \\
$m_{\tilde{\chi}_{2}^{ \pm}}[\mathrm{GeV}]$ & 105 & 107 & 105 & 105 & 107 & 105 \\
\hline$M_{W}[\mathrm{GeV}]$ & 80.427 & 80.422 & 80.422 & 80.432 & 80.430 & 80.427 \\
\hline$\chi^{2} /$ d.o.f. & $22.5 / 13$ & $22.2 / 13$ & $22.5 / 13$ & $14.2 / 13$ & $15.0 / 13$ & $15.0 / 13$ \\
\hline Probability & $4.8 \%$ & $5.2 \%$ & $4.8 \%$ & 32.8 & 35.9 & 30.5 \\
\hline
\end{tabular}

Table 2: The best fit parameters for different $\tan \beta$ scenarios are given. The masses are independent, i.e. they were choosen at low energies independent of possible GUT relations. All sparticle masses, which had no influence on the fit, like gluino, pseudoscalar mass, were set to a large value $(500 \mathrm{GeV})$. All squark masses were choosen to be equal, except for the stop masses. Also all slepton masses were choosen to be equal. In the last 3 columns the fit was repeated after rescaling the errors of $A_{F B}^{b}$ and $A^{L R}$ (see text). The chargino masses correspond to $\mu=135 \mathrm{GeV}$ and $M_{2}=170 \mathrm{GeV}$ in the notation of Ref. [1]. Note the increase in $M_{W}$ compared with the one in Table

\section{References}

[1] W. de Boer et al., Z. Phys. C75 (1997)627, hep-ph/9607286

[2] LEP Electroweak Working Group, LEPEWWG/2001-01, http://lepewwg.web.cern.ch/LEPEWWG/stanmod/

[3] H. N. Brown et al., hep-ex/0102017, Phys. Rev. Lett. 86 (2001) 2227.

[4] S. Narison, hep-ph/0103199, Phys. Lett. B513 (2001) 53.

[5] A. Czarnecki and W. Marciano, hep-ph/0102122.

[6] W. de Boer, M. Huber, C. Sander, D.I. Kazakov, Phys.Lett. B515 (2001) 283.

[7] CLEO Coll., S. Chen et al., CLEO 01/16, hep-ex/0108032;

[8] ALEPH Coll., R. Barate et al., Phys. Lett. B 429 (1998) 169.

[9] BELLE Coll., K.Abe et al., Phys. Lett. B 511 (2001) 151, hep-ex/0103042.

[10] P.Gambino and M.Misiak, Nucl. Phys. B611 (2001) 338, hep-ph/0104034.

[11] D. Bardin et al., Comput. Phys. Commun. 133 (2001) 229 and hep-ph/9908433; ZFITTER Version 6.10 was used.

[12] The LEP Collaborations, LEP Higgs Working Group, CERN-EP/2001-55, http://lephiggs.web.cern.ch/LEPHIGGS/papers/index.html 